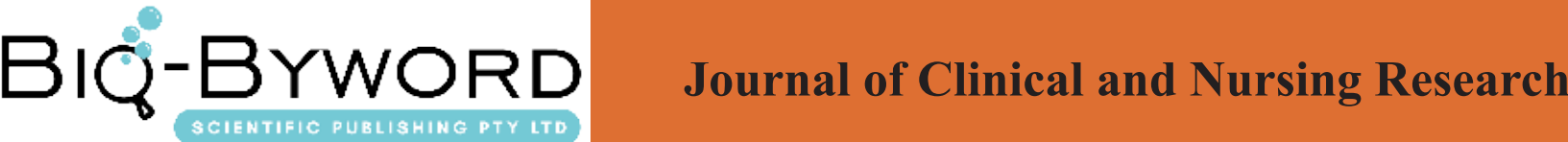

\section{Effect of Rehabilitation Training Combined with Psychological Intervention on Cognitive Function and Mental Behavior of Dementia Patients}

\author{
Xie Xiaolan He Xiangzhi Zhu Yanzhen* \\ Department of Neurology, the First Affiliated Hospital of Sun Yat-sen University, Guangzhou, Guangdong 510080
}

\section{ARTICLE INFO}

Article history:

Published online: 15th July, 2017

Key words:

rehabilitation training

joint psychological intervention dementia

cognitive function

mental behavior

\section{ABSTRACT}

Objective: To explore the effect of rehabilitation training combined with psychological intervention on cognitive function and mental behavior of dementia patients. Methods: A total of 142 patients with dementia from April 2015 to September 2016 were enrolled in this study. The patients were randomly divided into control group and experimental group. Among them, routine treatment was performed in the control group. In the control group, (MMSE) and daily living ability scale (ADL) were used to determine the MMSE and ADL scores of the two groups, and the patients were followed up for 6 months. Results: MMSE scores of the two groups were significantly higher than those before the intervention, and the MMSE score of the experimental group was significantly higher than that of the control group ( $\mathrm{P}<0.05)$. The ADL score of the two groups was significantly lower than that of the control group before and after intervention, the ADL score of the experimental group was significantly lower than that of the control group $(\mathrm{P}<0.05)$. Conclusion: Rehabilitation training combined with psychological intervention can effectively improve the cognitive function and mental behavior of dementia patients.

\footnotetext{
*Corresponding Author:
}

Zhu Yanzhen, Department of Neurology, the First Affiliated Hospital of Sun Yat-sen University, Guangzhou, Guangdong 510080. Email: feng_gzb@126.com 


\section{Introduction}

Dementia is a common clinical disease is also known as Alzheimer's disease, in the elderly population has a high risk of disease ${ }^{[1]}$. With the aging of our population, the incidence of dementia has gradually increased the trend. Dementia patients often appear memory loss, cognitive dysfunction and other symptoms, the patient's life have a serious impact ${ }^{[2-3]}$. There are no special effects of drugs for treatment, often in the clinical treatment of the way, although this method has a certain effect, but because of patients in the cognitive function and other specific aspects of the difference, the conventional way for many patients with limited effect. In this study, rehabilitation training combined with psychological intervention in dementia patients, and analysis of this method of dementia patients with cognitive and mental behavior, the specific study is as follows.

\subsection{General information}

Collected from our hospital in April 2015 to September 2016, 142 patients with dementia were analyzed, selected criteria ${ }^{[4-5]}$ : (1) Patients with significant memory loss, in terms of language, implementation and identification of obstacles; (2) All patients are in line with the American psychiatric AD diagnostic criteria. Exclusion criteria ${ }^{[6]}$ : (1) Patients with severe congenital mental retardation; (2) Patients with vision and hearing disorders. All patients and their families are informed and agreed to participate in this study. 142 patients with dementia were randomly divided into experimental group and control group of 71 cases, including 71 patients in the trial group, 36 males and 35 females, aged $60-83$ years, mean age $67.3 \pm 11.6$ years; course of $1-9$ years, the average duration of $5.3 \pm 1.6$ years; 16 cases of high school education, junior high school education in 24 cases, the following education in 31 cases of primary school. There were 71 patients, 37 males and 34 females, aged 61-84 years, with an average age of 68.2 \pm 12.1 years. There were 17 cases of high school education, 25 cases of junior high school education and 31 undergraduates. There was no significant difference between the two groups in the data. There was no significant difference between the two groups $(\mathrm{P}>0.05)$.

\subsection{Methods}

The control group: the patient for routine drug treatment, and the patient's normal treatment and basic daily life routine guidance.

The experimental group: on the basis of the control group of patients with rehabilitation training combined psychological intervention. Details as follows:

\section{(1) Cognitive function training}

According to the patient's specific condition and cognitive situation, by the medical staff for the patient to develop a reasonable training program, through the patient's interest in the way of guidance, such as organizing patients to organize activities in groups, etc., the patient's memory, judgment, understanding aspects of the cognitive function of training. Through the number, season, place, items and other aspects of the training of patients, training in accordance with the actual situation of the patient may be appropriate to increase the amount of training to promote the recovery of cognitive function of patients.

\section{(2) Daily life ability training}

Arrange the patients to carry out regular daily activities of the training to the basic eating, dressing, washing and other aspects of training as the basis, after several training gradually increase the content, such as finishing beds, simple cleaning and so on.

(3) Motor function training

According to the patient's condition for each patient for targeted exercise training, arrange for patients to carry out short-distance walking training, simple hand training, according to the actual situation of patients arranged training time. Organize patients in a small group of small game activities, through the form of game activities for training, the process of arranging special staff to guide, to maintain the rationality of training, and control the pace of training and time. For different hobbies of patients can arrange different types of training activities, training content to be rich and varied, including singing, playing chess, playing tai chi, drop, dance, walking and so on.

\section{(4) Language training}

Patients use language stories such as storytelling, speech reading, etc., encourage patients to communicate with each other, the process of nursing staff can be grouped through the organization of patients with centralized training for specific patients to conduct a oneon-one training alone.

\section{(5) Memory training}

The memory of the patient is trained by the memory text, the image and the item, and the sensation of the visual and auditory sensation of the patient is stimulated by repeated training to record the daily training of the 
patient, and the training of the patient is adjusted according to the training result.

\section{(6) Psychological intervention}

Nurses according to the patient's psychological state of psychological intervention in patients, psychological intervention process, the need to observe the patient's emotional changes, grasp the patient's mental state, for patients to eliminate doubts at the same time, positive to the patient to convey positive information to help patients build confidence.

(7) Training methods

All the training by the special care of the overall arrangements, and for different situations of patients to distinguish, adopt a step by step approach gradually, training time and methods in addition to follow the planning guidance, the nursing staff need to be adjusted according to the specific circumstances of the patient, training The specific details of the process can be based on the patient's point of interest and other reasonable improvements, different training programs can be an orderly combination.

\subsection{Evaluation indicators}

\subsubsection{Evaluation of cognitive function}

Using the Simple Mental State Examination Form
(MMSE) for Scoring, a total of 19 points, the score range of $0-30$ points, more than 24 points for the normal, the higher the score, the better cognitive function

1.3.2 Daily living ability and mental behavior assessment

Using the Daily Living Ability Scale (ADL) for scoring, with 14 item, the score range of 14-56 points, 16 points below the normal, the lower the score the daily living ability and mental behavior better.

\subsection{Statistical methods}

SPSS23.0 was used for processing. All data were normal distribution. The measurement results were expressed as mean \pm standard deviation $(\overline{\mathrm{x}} \pm \mathrm{s})$, and $\mathrm{t}$ test. The count data were expressed by the number of cases + percentile ( $\mathrm{n} \%$ ) and Chi-square test, if $\mathrm{P}<0.05$ indicates that the difference is statistically significant.

\section{Results}

2.1 Comparison of MMSE scores between the two groups

The results are shown in Table 1 . Table 1 shows that the MMSE scores of the two groups were significantly higher than those before the intervention, and the MMSE score of the experimental group was significantly higher than that of the control group $(\mathrm{P}<0.05)$.

Table 1 Comparison of MMSE scores in both groups ( $\overline{\mathrm{x}} \pm \mathrm{s}$, points)

\begin{tabular}{ccccc}
\hline Group & Before intervention & After intervention & t value & $\mathbf{P}$ \\
\hline Test group $(\mathbf{n}=\mathbf{7 1})$ & $13.9 \pm 1.6$ & $18.1 \pm 2.3$ & 12.631 & 0.000 \\
Control group $(\mathbf{n}=\mathbf{7 1})$ & $13.8 \pm 2.5$ & $15.1 \pm 1.7$ & 3.623 & 0.004 \\
$\mathbf{t}$ value & 0.284 & 8.838 & - & - \\
$\mathbf{P}$ & 0.777 & 0.000 & - & - \\
\hline
\end{tabular}

Table 1 Comparison of two groups of patients with ADL score ( $\mathrm{x} \pm \mathrm{s}$, points)

\begin{tabular}{ccccc}
\hline Group & Before intervention & After intervention & t value & P value \\
\hline Test group $(\mathbf{n}=\mathbf{7 1})$ & $31.7 \pm 9.3$ & $27.9 \pm 2.6$ & 3.316 & 0.001 \\
Control group $(\mathbf{n}=\mathbf{7 1})$ & $31.8 \pm 2.7$ & $29.6 \pm 5.1$ & 3.212 & 0.001 \\
$\mathbf{t}$ value & 0.087 & 2.502 & - & - \\
P value & 0.931 & 0.014 & - & - \\
\hline
\end{tabular}




\subsection{Comparison of ADL scores between the two groups}

The results are shown in Table 2. Table 2 shows that the ADL scores of the two groups were significantly lower than those before the intervention, and the ADL score in the intervention group was significantly lower than that in the control group $(\mathrm{P}<0.05)$.

\section{Discussion}

Dementia is a brain disease, dementia patients will appear memory loss, cognitive dysfunction and other phenomena, patients in personality and behavior are often affected ${ }^{[7]}$. Many factors that induce dementia, cerebrovascular disease, intracranial infection, cancer, metabolic diseases that affect brain function, etc. may lead to the occurrence of dementia. Dementia can have a serious impact on the health and life of the patient, causing a heavy burden on the patient's family ${ }^{[8]}$. At present, there is no special drug in the treatment of dementia, the treatment is very difficult treatment cycle is longer ${ }^{[9-10]}$, the clinical practice often used conventional treatment, poor treatment, the patient in the cognitive function and mental behavior to improve the effect is not obvious. In the course of treatment of patients, reasonable rehabilitation training and psychological intervention have an important role in the recovery of patients. In the study conducted by the hospital, patients were trained in systematic rehabilitation and combined with psychological intervention. Rehabilitation training includes cognitive function, daily life ability, motor function, language, memory and other aspects, each individual has a strong pertinence, different training between the complementary, such as cognitive function training and daily living ability training can be combined to improve cognitive function while improving the ability of daily life of patients and to optimize the quality of life of patients; language training and memory training is a single aspect of the patient to strengthen the project, to improve the patient's language skills and memory. Cognitive function also has a certain improvement, the same cognitive function in the training can also improve the patient's language, memory, intelligence and other aspects of the ability ${ }^{[11-13]}$. In addition to rehabilitation training for patients outside the psychological intervention in the treatment of patients also has a very important role in the care of patients in the process of psychological intervention, but also need to actively communicate with the families of patients to eliminate the concerns of family members, so that patient families are better able to participate in the treatment of patients. Many reports are aimed at patients with different items of training but less for the use of psychological intervention.
The study shows that rehabilitation training has a significant effect on cognitive function and daily living ability of dementia patients. Guo Dongmei ${ }^{[14]}$ and others stated in the rehabilitation of patients with Alzheimer's disease cognitive function, quality of life and depression in the study found that rehabilitation training can significantly improve the cognitive function and quality of life in patients with Alzheimer's disease. Zhao Guodong ${ }^{[15]}$ and others stated cognitive function training combined with daily life ability training in patients with Alzheimer's disease in the study found that cognitive function training combined with daily life ability training can improve the cognitive function of dementia patients and daily living ability. The results of this study also show that systematic rehabilitation training combined with psychological intervention can effectively improve the cognitive function and daily living ability of dementia patients.

In summary, rehabilitation training combined with psychological intervention can effectively improve the cognitive function and mental behavior of dementia patients, it is worth promoting the application.

\section{References:}

[1] Liu Jinyu, Xiao Lu, Wu Jimei. Rehabilitation Training On Senile Dementia Patients' Cognitive Function [J]. Chinese Journal of Practical Neurology, 2013, 16 (12): 24-25.

[2] Tan Xiaoxue, Luo Yuling, Zhao Li Ao, et al. Rehabilitation Training For Early And Middle-Aged Patients With Senile Dementia Cognitive Dysfunction [J]. Chinese Journal of Gerontology, 2012, 32 (18): 3876-3878.

[3] Wang Huifang. Rehabilitation Training on Senile Dementia Patients Cognitive Function Analysis [J]. Chinese Journal of Continuing Education, 2016, 8 (33): 233-235.

[4] Zhang Yaohong, Su Lin. System Nursing Intervention in Community Dementia Patients in the Application [J]. Nursing Research, 2015, 29 (8): 3008-3009.

[5] Shao Jing. Early Family Nursing Intervention on Senile Dementia Patients with Mild Cognitive Dysfunction [J]. Journal of Practical Medicine, 2012, 28 (21): 3654-3656.

[6] Liu Jiafeng. Memory Rehabilitation Training On Senile Dementia Patients with Cognitive Function Recovery [J]. Chinese community physician, 2016, 32 (20): 173-175. 
[7] Li Chunli, Chen Yan. Nursing Intervention on the Quality Of Life of Patients with Alzheimer's disease Analysis [J]. Anhui Medicine, 2014, 18 (6): 1182-1184.

[8] Liu Xiaoqin, Xie Lei. Rehabilitation Training In Patients with Senile Dementia Functional Independence and Cognitive Function of the Impact [J]. Practical Journal of Clinical Medicine, 2013, 10 (1): 75-76.

[9] Wang Jianan, Gao Jianhong. Effect of Personalized Nursing Intervention on the Quality of Dementia Patients [J]. Chinese Journal of Primary Medicine and Pharmacy, 2012, 19 (11): 1751-1752.

[10] Pan Yue, Yang Nan, Wang Ying. Family Members of Psychological Intervention in the Elderly Patients with Dementia in the Application of Nursing [J]. Chinese Journal of Clinical Health, 2012, 15 (2): 208-210.

[11] Hu Jue. Comprehensive Nursing Intervention in Patients with Alzheimer's disease And the Effect of
Observation [J]. Qilu Nursing Journal, 2012, 18 (22): 36-37.

[12] Zhang Yan, Liu Lei, Yan Mei .Elderly Patients with Dementia Cognitive Function Training Effect [J]. Chinese community physician, 2016, 32 (2): 154-155.

[13] Wang Hengfei, Chen Liping. Effect of Cognitive Behavior Nursing Intervention on Psychological Status of Dementia Patients [J]. Nurses Journal, 2014, 29 (2): 156-157.

[14] Guo Dongmei. Rehabilitation Training In Patients with Alzheimer's disease Cognitive Function, Quality Of Life And Depression [J]. Qilu Nursing Journal, 2016, 22 (1): 26-28.

[15] Zhao Guodong, Gao Mingming. Cognitive Function Training Combined with Daily Life Ability Training in Patients with Alzheimer's disease [J]. Chinese Health Standards Management, 2016, 7 (7): 60-61. 